\title{
The Effects of the Anion Clothes on Human Body Change in Young Adults
}

\author{
JongHun $\mathrm{Im}^{1}$, TaeGil Park ${ }^{2}$, Steve Goh ${ }^{3}$, JaeHo $\mathrm{Yu}^{{ }^{*} 1}$ \\ $*^{1}$ Department of Physical Therapy, Sunmoon University, ${ }^{2}$ GMK CO.,LTD, ${ }^{3}$ ENERSKIN Inc.
}

\begin{abstract}
Purpose The purpose of this study was to investigate the comparison of body changes (body temperature, grip power, forced vital capacity) according to pre-post comparative the anion clothes of young adults. Methods Forty young adults participated in this study. Each measurement was measuring 3 times. First is no wearing anion clothes, second measurement were after 10 minutes of wearing anion clothes and third measurement were after 30 minutes of wearing anion clothes. The body temperature measured using the infrared thermography. The area where the body temperature was measured were the neck, elbow, hand and calf. The grip force measured using the dynamometer. And the FVC measured using the Spirometry. All human body changes were analyzed by paired t-test. Significance probability of all statistics were set as $p<.05$. Results As shown in the results of measuring the grip power of the hand, there were significant difference between first measurement and third measurement, second measurement and third measurement. Discussion The FVC has significant difference all the measurement. And the body temperature were significant difference between first measurement and second measurement. There is no significant difference between first measurement and third measurement at neck because plateau phenomenon to maintain neck temperature. Anion has oxygen free radical so young adults clothing on anion has increased grip power, FVC and blood circulation also has improved.
\end{abstract}

Key words anion, clothes, body temperature, grip power, forced vital capacity,

Corresponding author JaeHo Yu (naresa@sunmoon.ac.kr)

Received date 22 January 2018

Revised date 7 February 2018

Accepted date 21 February 2018

\section{Introduction}

Ions are sketched when electrons separated from the molecules of the gas have sufficient external energy. When an electron is separated from a molecule, it becomes a cation, while free electrons stick to an adjacent molecule to form an anion. ${ }^{1)}$ Anion is a molecule with a negative charge in the atmosphere, which promotes human body fatigue and metabolism. ${ }^{2}$ There have been many studies on the effects of anions on humans, but they failed to show the objective effects of negative ions. ${ }^{3)}$ Also, anions suppress serotonin hypersecretion and promote cell metabolism. ${ }^{4}$ Studies by Ryushi have shown that anions significantly reduce blood pressure and heart rate during and after exercise. ${ }^{5}$ These studies have shown that anions contribute to the body's positive effects and blood

doi : http:dx.doi.org/10.17817/2018.01.26.111237 circulation. When the anion is inhaled into the body, the binding of red blood cells falls and the intake of oxygen is improved. ${ }^{6}$ Because of the effect of these anions on the human body, there is a tendency for the forests to release large amounts of anion in order to find anion in modern society.

According to Suzuki's study, when anion is exposed to large amounts, sympathetic activity decreases and parasympathetic nerves become active. ${ }^{7)}$ The sympathetic nervous system is responsible for up and down regulating homeostatic mechanisms in living organisms like increases rate and force of contraction of the heart, Dilates in skeletal muscles with blood vessels and promotes emission prior to ejaculation. ${ }^{8)}$ Also the sympathetic nervous system is responsible for activating the body function for action." Whereas parasympathetic nerves system affects many part of the body like the heart relaxes and help make the blood 
pressure lower. ${ }^{10)}$ Therefore, as mentioned above, anions not only have a positive effect on the human body in daily life, but also activate the parasympathetic nerves when exercising to restore the human body after exercise.

Based on the study on the effects of the above anions on human body, this study aimed to experiment the effects of the anions. Negative ion clothing is used to detect the change of the human body temperature, the grip power, and the change of the forced vital capacity, and to allow easy exposure to negative ions to enable better daily life. Through this study, I tried to find out whether the negative recovery of the human body when wearing anion clothes and the quick recovery when exercising.

\section{Materials and Methods}

\section{Research subjects}

The subject of this research were the young adults of $S$ University at Asan, Chungnam. Subjects were selected with 20 males and 20 females, so total 40 adults. Plenty of education regarding the research purposes, methods were conducted before the experiment. The research subjects were those who had agreed to research participation, and exclusion criteria of this study were 1) orthopedic, neurological surgical problems when they get the grip 2) in the last six months of surgery or affecting the holding operation and 3) cardiovascular disease. Also, total of 40 research subjects were selected by utilizing G*Power3.1.

\section{Experimental procedure}

The whole process of the experiment is shown in (Figure 1). The subjects measured body temperature(neck, elbow, hand and calf), FVC(forced vital capacity), and grip power to determine the change of body. The each measurement were measuring 3 times. First measurement; no wearing anion clothes, second measurement; after 10 minutes of wearing anion clothes and third measurement; after 30 minutes of wearing anion clothes(after 20 minute exercise)(Figure 2). Anion clothes was pressurized at a pressure of 45
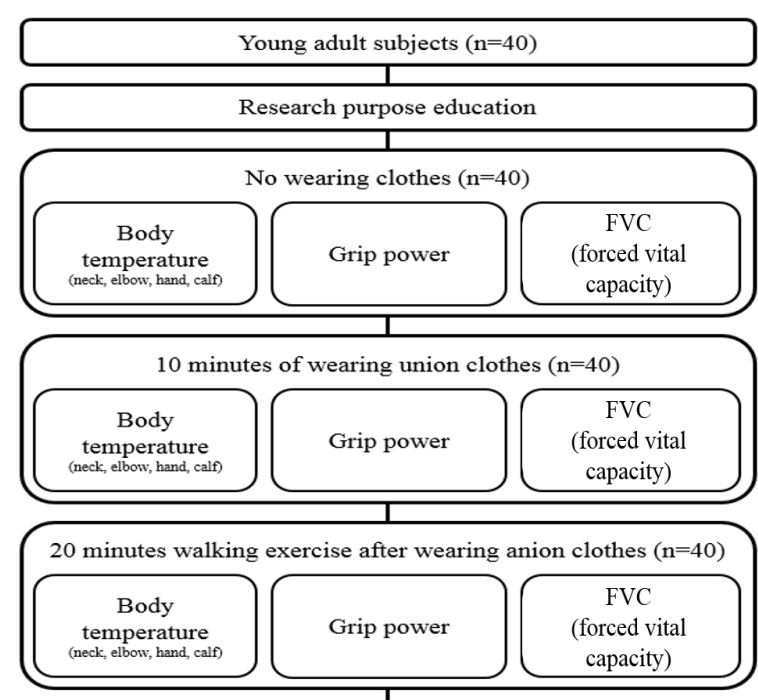

Data analysis $(n=40)$

Figure 1. Research process

$\sim 55$ PSI(pound per square inch) at a temperature of $170 \sim 180$ degree for $9 \sim 13$ seconds to make a silicone patch. The anion concentration of the patch was set at 1500 , and the patch was applied to the subjects. The results were averaged after 3 measurements. Subjects were rested for 10 minutes after first measurement until second measurements. Subjects were walked for 20 minutes, up to third measurements after second measurements.

\subsection{Body Temperature}

Body temperature were measured using the infrared thermography. Body temperature was set to reach the whole body. The area of the temperature measurement were neck, elbow, hand and calf to measure change of body temperature. The subjects stood in front of the infrared thermography and looked straight ahead and standing anatomical posture. As mentioned in the experimental procedure, they were measured 3 times 1) before wearing anion clothes, 2) 10 minutes after wearing anion clothes and 30 minutes after wearing anion clothes, measuring 3 times each time, and the results were averaged(Figure 3).

\subsection{Grip Power}

Grip power were measured using the dynamometer. The subjects were in a standing posture with flexion 
elbows 30 degrees and grasped the grip force with maximum force using the dominant hand. As mentioned in the experimental procedure, they were measured 3 times 1) before wearing anion clothes, 2) 10 minutes after wearing anion clothes and $30 \mathrm{mi}-$ nutes after wearing anion clothes, measuring 3 times each time, and the results were averaged(Figure 4).

\subsection{FVC}

FVC were measured using the spirometry. The subjects performed deep breathing three times in a standing posture and then breathed a deep breath and measured the FVC when spitting the maximum amount of breath using a spirometry. As mentioned in the experimental procedure, they were measured 3 times 1) before wearing anion clothes, 2) 10 minutes after wearing anion clothes and 30 minutes after wearing anion clothes, measuring 3 times each time, and the results were averaged(Figure 5).

\section{Data Analysis}

Statistical analysis was performed using SPSS version 22.0 for Window (SPSS Inc., Chicago, IL, USA). The
Table 1. General characteristics of the subjects ( $n=40,20$ men and 20women)

\begin{tabular}{lc} 
& participation \\
Age (years) & $20.9 \pm 2.34$ \\
Height $(\mathrm{cm})$ & $167.78 \pm 7.51$ \\
Weight $(\mathrm{kg})$ & $63.5 \pm 13.74$ \\
\hline
\end{tabular}

Mean \pm Standard deviation

mean and standard deviation of the variables were calculated by descriptive statistics and paired t-test(Wilcoxon signed-rank test) was used to compare the change of the human body; body temperature, grip power, FVC. The level of statistical significance was set at $\mathrm{p}<.05$.

\section{Results}

A total of 40 young adults (20men and 20women) participated in this study. The mean age of the subjects was 20.9 years, mean height was $167.78 \mathrm{~cm}$, and mean weight was $63.5 \mathrm{~kg}$ (Table 1$)$.

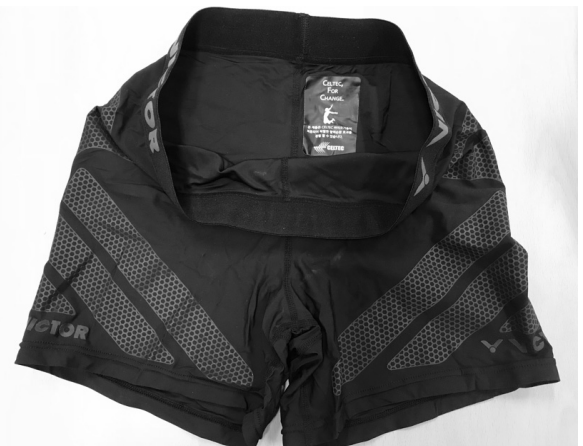

Figure 2. anion clothes

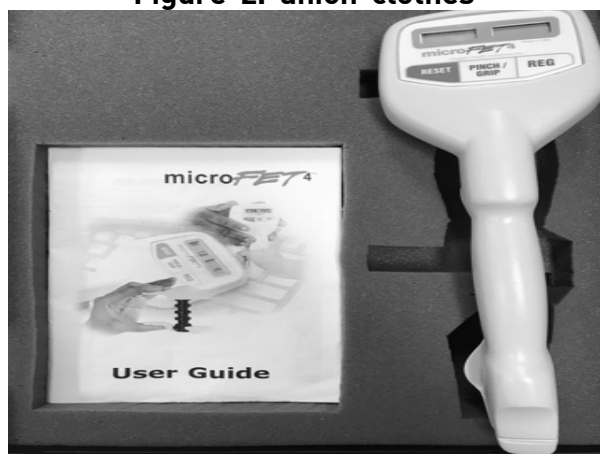

Figure 4. dynamometer

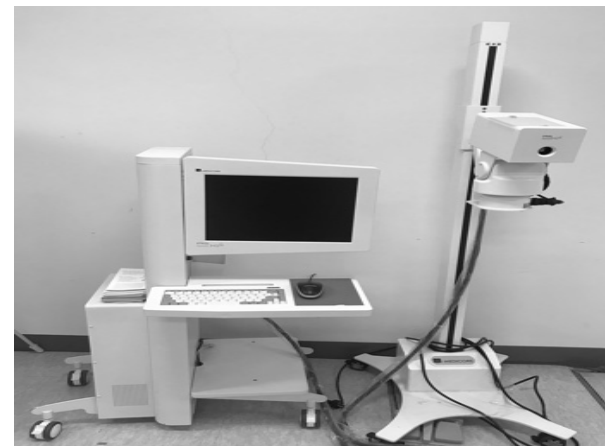

Figure 3. infrared thermography

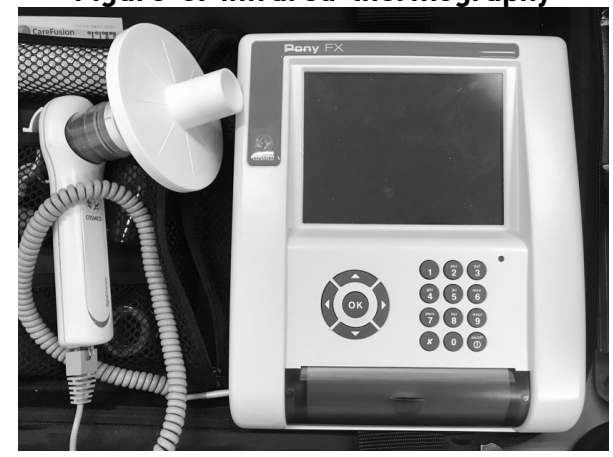

Figure 5. spirometry 
Table 2. Measurement of each body ability

\begin{tabular}{ccccc}
\hline & & first measurement & second measurement & third measurement \\
body & neck & $29.99 \pm 3.5$ & $30.72 \pm 2.67$ & $30.38 \pm 2.53$ \\
temperature $\left({ }^{\circ} \mathrm{C}\right)$ & elbow & $29.26 \pm 3.09$ & $29.59 \pm 2.71$ & $29.07 \pm 2.27$ \\
& hand & $26.8 \pm 4.06$ & $27.72 \pm 2.93$ & $28.68 \pm 2.57$ \\
calf & $27.87 \pm 3.6$ & $28.28 \pm 2.67$ & $28.82 \pm 2.55$ \\
Grip power (kg) & $79.77 \pm 20.67$ & $80.29 \pm 21.59$ & $83.42 \pm 22.06$ \\
\multicolumn{2}{c}{ FVC(\%) } & $68.47 \pm 9.68$ & $69.97 \pm 10.63$ & $71.47 \pm 10.8$ \\
\hline
\end{tabular}

Mean \pm Standard deviation, first measurement : before wearing anion clothes, second measurement : After 10 minutes of wearing anion clothes, third measurement : After 30 minutes of wearing anion clothes (after 20 minute exercise)

The results of each measurement(The first, the second and the third) showed some increase. In the temperature $\left({ }^{\circ} \mathrm{C}\right)$ : the first measurement of the neck part was $29.99 \pm 3.50$, the second measurement was $30.72 \pm 2.67$ and the third measurement was $30.38 \pm 2.53$. Between first measurement and second measurement showed significant differences $(p<.05)$, but between first measurement and third measurement, second measurement and third measurement did not show significant differences. The first measurement of the elbow was $29.26 \pm 3.09$, the second measurement was $29.59 \pm 2.71$ and the third measurement was $29.07 \pm 2.27$. Between first measurement and second measurement, second measurement and third measurement, first measurement and third measurement did not show significant differences. The first measurement of the hand was 26.8 \pm 4.06 , the second measurement was $7.72 \pm 2.93$ and the third measurement was $28.68 \pm 2.57$. Between first measurement and second measurement, second measurement and third measurement, first measurement and third measurement showed significant differences $(p<.05)$. The first measurement of the knee was $27.87 \pm 3.6$, the second measurement was $28.28 \pm 2.67$ and the third measurement was $28.82 \pm 2.55$. Between first measurement and second measurement, second measurement and third measurement showed significant differences $(p<.05)$, but between first measurement and third measurement did not show significant differences. In the grip power(kg); the first measurement was $79.77 \pm 20.67$, second part was $80.29 \pm 21.59$ and third part was $83.42 \pm 22.06$. Between first measure- ment and third measurement, second measurement and third measurement showed significant differences $(p<.05)$, but between first measurement and second measurement did not show significant differences. Finally, in the FVC(\%) showed an increase in respiratory function in the first part was $68.47 \pm 9.68$, second part was $69.97 \pm 10.63$ and third part was $71.47 \pm 10.8$ (Table 2). Between first measurement and second measurement, second measurement and third measurement, first measurement and third measurement showed significant differences $(p<.05)$ (Table 3 ).

\section{Discussion}

The purpose of this study was to investigate changes in body temperature, grip power, and FVC of the subjects by using anion clothes and measuring the total of 3 times before, 10 minutes after wearing and 30 minutes after wearing anion clothes. According to the results of the study, the overall body temperature, grip power and FVC were increased before and after anion clothing.

According to the results of the body temperature measurement, the temperature increased before and 30 minutes after wearing the elbows and neck, but there was no significant difference between before and after exercise. On the other hand, the temperature of the hand and calf increased with time and there was a significant difference during the each measurements. According to Edyta's study, blood cir- 
Table 3. Difference of each times

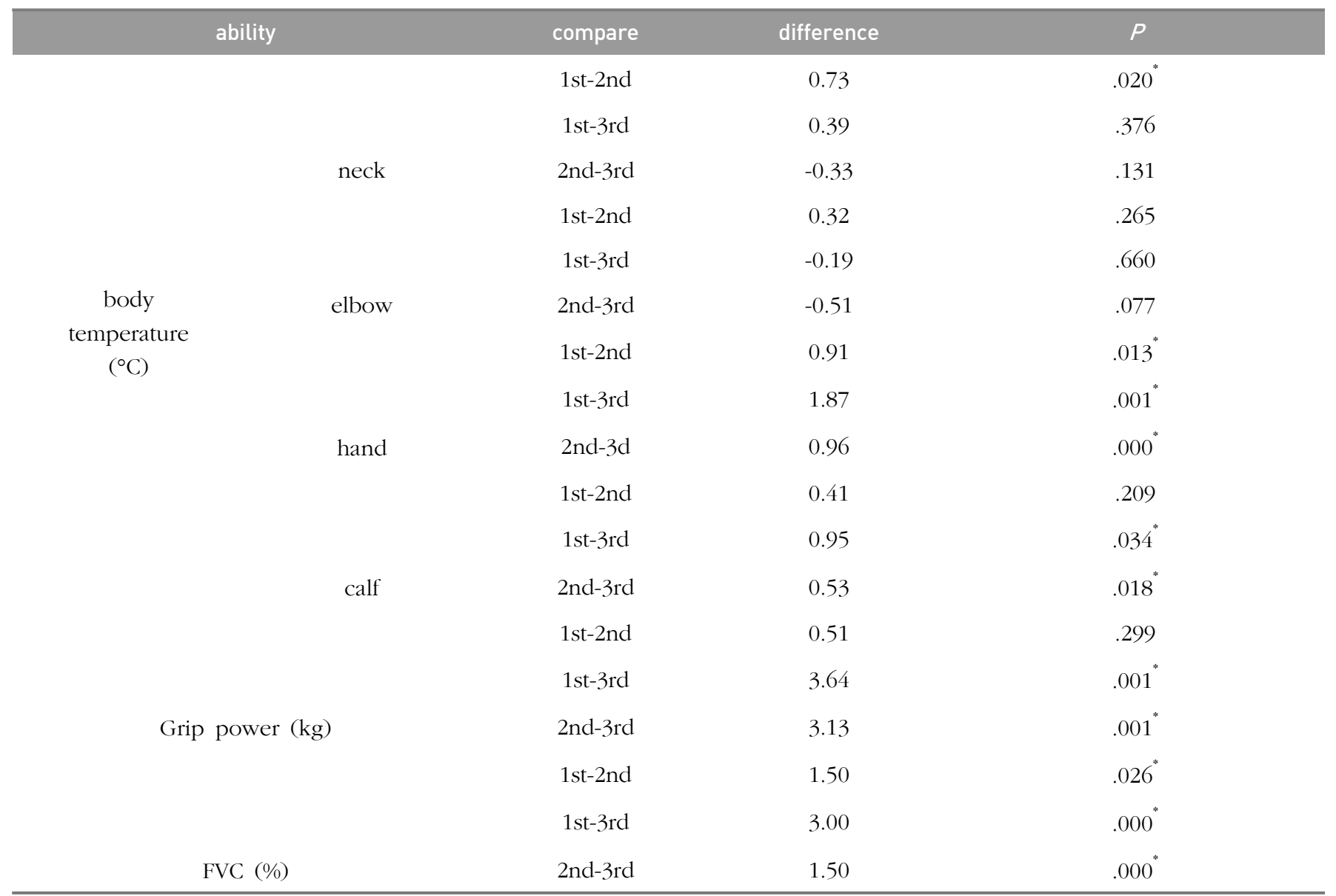

* Significant difference $(p<0.05), 1$ st-2nd : Difference between first measurement and second measurement, 1st-3rd : Difference between first measurement and third measurement, 2nd-3rd : Difference between second measurement and third measurement

culation is better because anion is separated from red blood cells when exposed to human body. ${ }^{11)}$ Therefore, the body heat measurement showed a significant difference in the body temperature after the anion was exposed at the portion excluding the elbow $(p<0.05)$. Also, there was a significant difference in the arm and legs, which were distal part from the body when wearing and before exercising with anion clothes $(p<0.05)$. On the other hand, there was no significant difference in body heat between neck and elbow before and after exercise $(p>0.05)$. According to Ryushi's study, the effect of reducing the heart rate after exercising anions is due to the fact that kessel's study shows a platelet phenomenon that reduces excessive heart rate ${ }^{5,12}$. According to the results of this study, exposure of anions increases blood circulation in daily life and regulates excessive blood circulation when exercising.

According to Margherita's study, the subject's body temperature is increased and this heat helps to improve leg strength ${ }^{13)}$. According to edwards' study, when the subject's body temperature increased, muscle strength was increased, which was an increase in muscle metabolism ${ }^{14)}$. In the first measurement of this study, body temperature was increased by wearing anion clothes. In the second measurement, the grip power was increased. This can be supported by the results of this study, in which muscle power is increased because of increased metabolism of muscles as the body temperature is increased based on the above two articles.

According to Rho's study, oxidative activity after 
exercise was significantly higher in pre exercise than in pre exercise when oxygen and anion intervention had effects on oxidative activity at maximal exercise. ${ }^{15}$. Exercise increases reactive oxygen in the muscle by increasing the consumption of oxygen, and high intensity exercise increases antioxidant enzyme activity. ${ }^{16)}$ Based on the above study, this study can support the fact that when FVC is measured by obtaining active oxygen on the human body when wearing anion clothes, there is a significant difference in all pre-wear, pre-exercise, and post-exercise.

There are some limitations to this study. First, the subjects were not able to compare various age groups only by 20 ordinary adults. Second, when the body temperature was measured, the foot was not measured. Third, body temperature was measured on neck, elbow, hand and calf but muscle strength was only tested for grip power. Our study needs to study the correlation between body temperature and muscle strength by measuring muscle strength in various parts like body temperature.

In conclusion, forty young adults were totally effective when Comparing with the anion changes before and after the wearing of clothes, overall effect was. Especially, the effect of anions showed a significant difference in body temperature, as the body temperature increased, the change of the hand power was also significant. In addition, there was a significant difference in FVC due to the influx of active oxygen in anions. Therefore, anion clothes improves human body function, and when exercised, it regulates to the plate phenomenon normally.

\section{References}

1. Stefan M, Gudrun M. Involvement of the Superoxide Anion Radical in the Autoxidation of Pyrogallol and a Convenient Assay for Superoxide Dismutase. Eur J Biochem. 1974;47(3):496-74.

2. Nakane H, Asami O, Yamada $Y$, et al. Effect of negative air ions on computer operation, anxiety and salivary chromogranin A-like immunoreactivity. Int $\mathrm{J}$ Psychophysiol. 2002;46(1):85-9

3. Lee J, Jang M, Chae E, et al. Effects of the inhalation of negative air ions on heart rate variability. Korean J Fam Pract. 2017;7(2):253-57.

4. Vicrot RB. Behavioural effect of air lons. 1975.

5. Ryushi T, Kita I, Sakurai T, et al. The effect of exposure to negative air ions on the recovery of physiological responses after moderate endurance exercise. Int $\mathrm{J}$ Biometeorol. 1998;41(1):132-6.

6. Kim WK, Kim SS, Yu SH, et al. Influence of far-infrared rays and anions on human body. journal of oriental preventive medical society. 2005;9(2):93-106.

7. Suzuki S, Yanagita S, Amemiya S, et al. Effects of negative air ions on activity of neural substrates involved in autonomic regulation in rats. Int $\mathrm{J}$ Biometeorol. 2008;52(1):481-9.

8. Moro C, Tajouri L, Chess WR. Adrenoceptor function and expression in bladder urothelium and lamina propria. Urology. 2013;81(1):211-7.

9. Robert O. The evolution of consciousness: of Darwin, Freud, and Cranial Fire: The Origins of the Way We Think. New York: Simon \& Schuster. 1992.

10. Blakemore C, Jennett S. The Oxford Companion to the Body. Oxford University Press. 2001.

11. Edyta G, Agnieszka D, Anne C, et al. Anion conductance of the human red cell is carried by a maxi-anion channel. Blood Cells Mol Dis. 2010;44(4)243-51

12. Kessel L, Johnson L, Arvidsson H, et al. The relationship between body and ambient temperature and corneal temperature. Invest Ophthalmol Vis Sci. 2010;52(12): 6593-7.

13. McKay WP, Vargo M, Chilibeck PD, et al. Effects of ambient temperature on mechanomyography of resting quadriceps muscle. Appl Physiol Nutr Metab. 2013; 38(3):227-33.

14. Edwards RHT, Harris RC, Hultman E. Effect of temperature on muscle energy metabolism and endurance during successive isometric contractions, sustained to fatigue, of the quadriceps muscle in man. J Physiol. 1982;49(2):243-51.

15. Rho SK, Kim HL. Effects of $\mathrm{O} 2$ and minus ion treatment on oxidant and antioxidant enzymes activity during maximal GXT in men and women. The Asian Journal of Kinesiology. 2007;9(1):59-67

16. 16. Davies KJA, Quintanilha AT, Brooks GA, et al. Free radicals and tissue damage produced by exercise. Biochem Biophys Res Commun. 1982;107(4):1198-205 